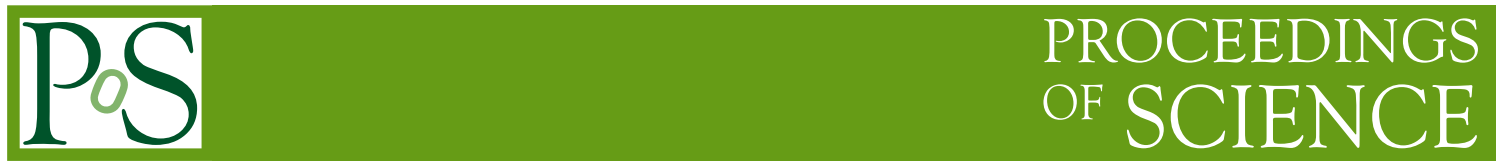

\title{
The relevance of jet emitting discs in micro-quasars
}

Pierre-Olivier Petrucci ${ }^{* \dagger}$

IPAG, Grenoble, France

E-mail: petrucci@obs.ujf-grenoble.fr

\section{Jonathan Ferreira, Gilles Henri}

IPAG, Grenoble, France

E-mail: ferreira@obs.ujf-grenoble.fr

\section{Clément Cabanac, Julien Malzac, Renaud Belmont}

IRAP, Toulouse, France

E-mail: clement.cabanacecesr.fr

Jet Emitting Disc (JED) has dynamical properties quite different from both the standard and advection dominated discs. It also exhibits three different thermal equilibrium branches at a given radius: two stable (cold and hot) and one intermediate unstable. The hot solution has all the characteristics of the so-called "hot corona" generally invoked in XrB sytems in the Low/Hard states. We detail the energetics and radiative expectations of our model and show their good agreement with those observed in Cygnus X-1 in terms of jet power, jet velocity and spectral emission.

Fast $X$-ray timing and spectroscopy at extreme count rates

February 7-11, 2011

Champéry, Switzerland

\footnotetext{
* Speaker.

${ }^{\dagger}$ PoP acknowledges financial support from the CNES and from the french GDR PCHE.
} 


\section{Jet Emitting Dises}

Self-confined jets are observed around a wide variety of astrophysical objects, namely from young forming stars (YSOs), neutron stars or stellar black holes (X-ray binaries) and supermassive black holes (AGN). Although the underlying emission mechanisms are quite different (molecular and atomic lines in YSOs, synchrotron radiation from supra-thermal particles in LMXBs and AGN), they share common properties: a high degree of collimation (opening angle of a few degrees), a systematic link with the accretion disc and carry away a sizable fraction of the released accretion power. It is thus natural to seek for a model where accretion and ejection processes are interdependent regardless of the central object (be it a star or a compact object).

The most promising and so far developed model is ejection from a quasi-Keplerian accretion disc, commonly referred to Blandford \& Payne (2) jets (hereafter BP). In this model, a large scale magnetic field of bipolar topology is assumed to thread a near-Keplerian accretion disc. This field extracts angular momentum and energy from the disc and transfers them back to the ejected material. This material is then accelerated to infinity and maintained self-confined by the hoopstress due to the strong toroidal field. This self-confinement effect has been shown by (2) and discussed by many other subsequent authors. However, what remained to be done was to compute the connection with the underlying disc, since the BP model was using ad-hoc boundary conditions at the disc surface.

This work has been done more than 10 years ago by $(10 ; 11 ; 8)$. Using a self-similar ansatz, it is indeed possible to solve exactly the full set of non-relativistic MHD equations describing a resistive, viscous accretion disc thread by a large scale field. Both viscosity $v_{v}$ and magnetic diffusivity $v_{m}$ are assumed to arise from a MHD turbulence and were modeled with an alphaprescription, namely $v_{m}=\alpha_{m} V_{A} h$ where $V_{A}$ is the Alfvén velocity on the disc midplane, $h$ the disc vertical scale height and $v_{v}=\mathscr{P}_{m} v_{m}$ where $\mathscr{P}_{m}$ is the effective magnetic Prandtl number. The set of MHD equations have been solved from the disc midplane to the jet termination point, subjected to the constraint of smoothly crossing the critical points of the flow (mainly slow-magnetosonic and Alfvén). These two regularity conditions are unbiased by the self-similar ansatz and severely constrain the parameter space of these Jet Emitting Discs (hereafter JEDs). Indeed, defining the disc magnetization $\mu=B_{z}^{2} / P$ where $P=P_{g a s}+P_{r a d}$, it has been shown that powerful jets can only be obtained with $0.1<\mu<1$, namely a large scale field close to equipartition. This result was in severe contrast with previous works (e.g. $(15 ; 16))$ and explains why the latter could not obtain super-Alfvenic, steady-state jets from thin discs. Solutions linked to the disc and crossing the three MHD critical points were also obtained, but it was shown that self-similarity introduces a strong bias on the fast-magnetosonic point that is probably unphysical (9).

Because of the mass loss, the disc accretion rate varies as $\dot{M}_{a} \propto r^{\xi}$, where $\xi$ is the disc ejection efficiency: the higher $\xi$ the more mass is being loaded onto the jets. Models with isothermal or adiabatic magnetic surfaces achieve typical values of $\xi \sim 0.01$ (6), whereas more heavily loaded jets with $\xi \sim 0.1$ can be obtained when a surface heating is present (7). The asymptotic velocity reached along a given field line is a direct function of $\xi$. In a non-relativistic jet, it writes $v_{j}=$ $v_{K} \sqrt{2 \lambda-3}$, where $\lambda \simeq 1+\frac{1}{2 \xi}$ is the magnetic lever arm and $v_{K}$ the Keplerian speed at the line footpoint. Thus, contrary to the usual belief, jets from near-Keplerian accretion discs can have velocities much larger than $v_{K}$. However, because the mass loss is not that tiny, highly relativistic 


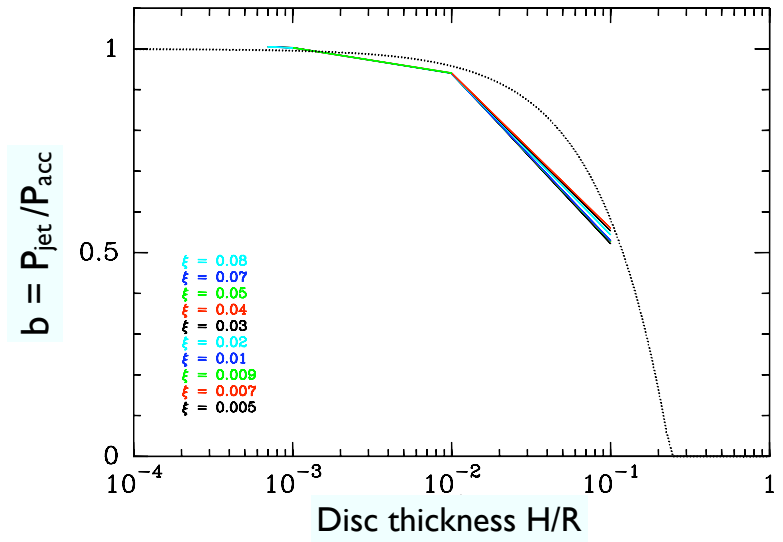

Figure 1: Ratio of the total jet power to the total accretion power as a function of the disc thickness, for all isothermal models of (8). Note that the power decreases rapidly, regardless of the value of the disc ejection efficiency $\xi$.

jets are also difficult. Indeed, the jet magnetization, namely the ratio of the MHD Poynting flux to the kinetic energy flux at the disc surface is $\sigma \simeq \xi^{-1}$ and never reaches the high values $\left(10^{4}\right.$ to $\left.10^{6}\right)$ sometimes invoked. As a consequence, only mildly relativistic flows are expected, but not flows with bulk Lorentz factors around 10 or 20. For those, another mechanism must be found, either extracting energy from a rotating black hole (3) or a two-component jet (see e.g. (4) and references therein).

Moreover, (6) remarked that they could not find any steady-state JED solution with a disc aspect ratio $\varepsilon=h / r>0.3$. This is because, as the disc gets thicker, the effect of the radial magnetic tension increases drastically so as to cancel rotation at the disc surface. As a consequence, the toroidal field vanishes and so does the MHD Poynting flux: there is no more power to feed the jets. Figure (1) shows the total power $P_{\text {jets }}$ (mechanical, thermal and electromagnetic) leaving the disc normalized to the accretion power $P_{a c c}$ as a function of the disc aspect ratio $h / r$ (see (12)). Geometrically thin discs give rise to jets that carry away up to $99 \%$ of the released accretion power whereas slim discs with $\varepsilon=0.1$ can only provide $50 \%$ to their jets. This power goes rapidly to zero so that no super-Alfvénic isothermal jet can be found from thick discs with $\varepsilon=h / r>0.3$. Hence, ADAFs are unable to drive powerful jets, although thermally driven winds would be a natural outcome (ADIOS, (1)). Global MHD simulations do show ejection from thick disc structures, but they are probably of this kind, carrying a small fraction only of the released power. On the other hand, 2.5D MHD simulations of alpha discs recover all of the results given by self-similar solutions (see (19) and references therein).

\section{Comparison to Cygnus X-1}

The resolution of the thermal equilibrium of a JED gives three branches, a cold and hot ones, both thermally and viscously stable and an intermediate unstable one. The hot branch, that would be observationally very similar to ADAFs, corresponds to the JED solution discussed below and applied to Cyg. X-1 (17). Thus JEDs could account for most of the successes of the ADAFs, while explaining jet formation. 

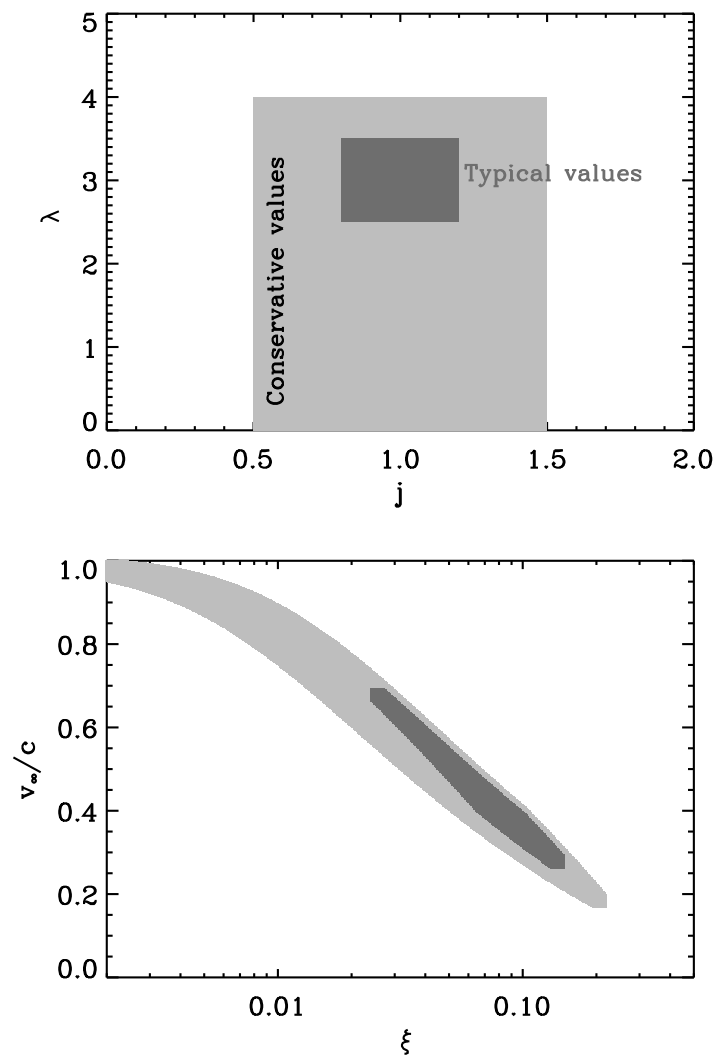

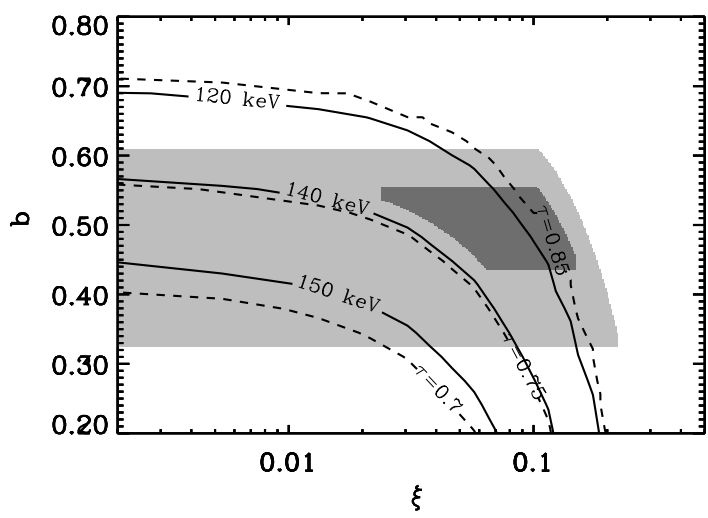

Figure 2: Top-left: the parameter space $j-\lambda$ allowed by the observations. Top right: Observational constraints translated into our JED parameter space $\xi-b$. Overplotted are the contours of temperature (solid lines) and optical depth (dashed lines) for an accretion rate of 1-2\% Edd., typical of Cyg X-1. Bottom-left: terminal jet velocity $V_{\infty} / c$ as function of the disc ejection efficiency $\xi$

The energetics of the jets and the X-ray corona of Cygnus X-1 have been investigated recently by Malzac et al. (2009 herafter M09). Observations constraint the ratio $j=P_{\text {jets }} / L_{h}$ of the total jet kinetic power to the typical X-ray luminosity in the hard state as well as the ratio $\lambda$ of the soft to hard radiative efficiencies to be in the ranges (M09):

$$
0.45 \leq j=\frac{P_{j e t s}}{L_{h}} \leq 1.5 \text { and } \lambda=\frac{L_{s}}{\dot{M}_{s}} \frac{\dot{M}_{h}}{L_{h}} \leq 4
$$

These observational constraints on $j$ and $\lambda$ can be easily translated into constraints on our JED parameters $b$ and $\xi$, where $P_{\text {jets }} \simeq b P_{a c c}$ and $\dot{M}_{a}(R) \propto R^{\xi}$. The top-left panel of Fig. (2) displays the domain in the observed parameter space $j-\lambda$ allowed by the observations. The top right panel shows the same constraints but translated into our JED parameter space $\xi-b$. Overplotted are the contours of the JED temperature and optical depth for an accretion rate of 1-2\% Edd., typical of Cyg X-1. The bottom-left panel of Fig. (2) shows the terminal jet velocity $V_{\infty} / c$ as function of the disc ejection efficiency $\xi$ which are in good agreement with observations.

The accretion and ejection properties of JEDs agree with the observations of the prototypical black hole binary Cygnus X-1. The JED solutions are likely to be relevant to the whole class of microquasars.

\section{A toy model for the $\mathrm{X}$-ray variability in the Hard state}

In the LHS the X-ray corona is generally assumed to be localized at the jet basis, producing 

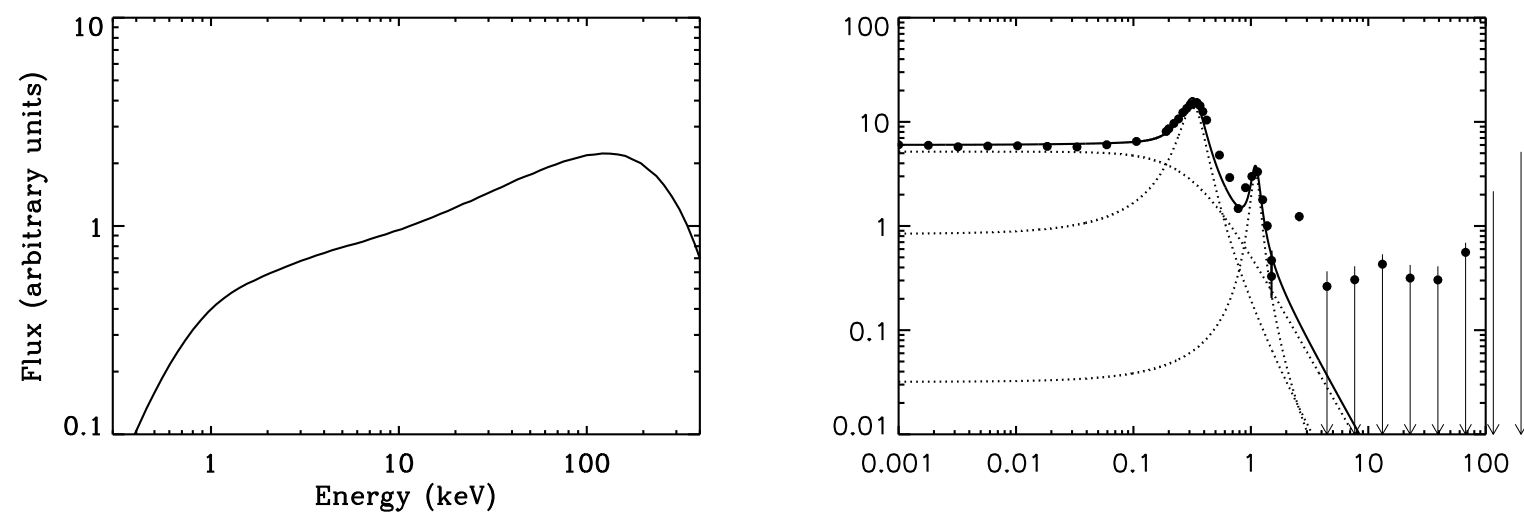

Figure 3: Simulated Monte Carlo SED and PDS expected from a cylindical corona with mean optical depth and temperature $\tau=1.4$ and $k T_{e}=100 \mathrm{keV}$ when this corona is excited by a external white noise.

$\mathrm{X}$-rays through comptonisation of surrounding soft photons. In our view, the jet basis looks like a cylinder with $r_{i n}$ and $r_{t r}$ as the inner and outer radii respectively. The outcoming spectrum then depends on the optical depth and temperature of this corona. The observed spectra in the hard state generally required $\tau \sim 1$ and $k T_{e} \sim 100 \mathrm{keV}$. We propose that the observed variability in this state is produce by the oscillation of the corona through external excitation in $r_{t r}$ producing compressive waves in the corona. This idea was already proposed by (14) and we study this effect through Monte-Carlo simulations. Perturbations $\partial n(r, t)$ and $\partial T(r, t)$ of the corona density and temperature then produce spectral variability in the X-ray spectra. For low frequency oscillations, the density and temperature perturbation have the same sign in the corona. Thus we expect large effects on the output spectrum i.e. large rms. On the contrary, for high frequency oscillations, $\partial n(r, t)$ and $\partial T(r, t)$ change sign between $r_{i n}$ and $r_{t r}$. In this case, multiple compton scatterings smear the spectral variability effects on the outcoming spectrum. The larger the oscillation frequency the weaker the variability and the smaller the rms. Now, depending on the boundary conditions, resonances where $\partial n(r, t)$ and $\partial T(r, t)$ reaches large amplitude may exist producing QPOs in the Power Density Spectrum (PDS). We show in Fig. 3 the simulated Monte Carlo SED and PDS expected from a cylindical corona with mean optical depth and temperature $\tau=1.4$ and $k T_{e}=100 \mathrm{keV}$ when this corona is excited by a external white noise and when $r_{i n}$ and $r_{t r}$ are nodes of the compressive waves. The SED looks very similar to the common LHS SED. The PDS show a flat top profile at low frequency, decreasing at high frequency (above $\sim\left(k T / m_{p}\right)^{0.5} / r_{t r}$ ), in agreement with the PDS observed in this state. However different QPOs, that correspond to the different resonances of the system are also present. While low frequency QPOs are known to be present in the hard state/High intermediate state, close to the cut-off frequency, the presence of multiple low frequency QPOs like in our simulations are rather uncommon (but see (18)). The powers of these peaks depend on the boundary conditions chosen (in he present case, we assume a "total wave reflection" at the inner corona radius $r_{i} n$ ) and, more specifically, on the impedance adaptation with the external medium at the corona inner boundary (5).

\section{References}

[1] Blandford, R. D. \& Begelman, M. C. 1999, MNRAS, 303, L1 
[2] Blandford, R. D. \& Payne, D. G. 1982, MNRAS, 199, 883

[3] Blandford, R. D. \& Znajek, R. L. 1977, MNRAS, 179, 433

[4] Boutelier, T., Henri, G., \& Petrucci, P. 2008, MNRAS, 390, L73

[5] Cabanac, C., et al., \& Belloni, T. M. 2010, MNRAS, 404, 738

[6] Casse, F. \& Ferreira, J. 2000a, A\&A, 353, 1115

[7] Casse, F. \& Ferreira, J. 2000b, $A \& A, 361,1178$

[8] Ferreira, J. 1997, $A \& A, 319,340$

[9] Ferreira, J. \& Casse, F. 2004, ApJL, 601, L139

[10] Ferreira, J. \& Pelletier, G. 1993, A\&A, 276, 625

[11] Ferreira, J. \& Pelletier, G. 1995, A\&A, 295, 807

[12] Ferreira, J., \& Petrucci, P. O. 2011, IAU Symposium, 275, 260

[13] Malzac, J., Belmont, R., \& Fabian, A. C. 2009, MNRAS, 400, 1512

[14] R. Misra, 2000 ApJL 529, L95-L98

[15] Ogilvie, G. I. \& Livio, M. 1998, ApJ, 499, 329

[16] Ogilvie, G. I. \& Livio, M. 2001, ApJ, 553, 158

[17] Petrucci, P.-O., Ferreira, J., Henri, G., Malzac, J., \& Foellmi, C. 2010, A\&A, 522, A38

[18] J. Rodriguez, et al. 2004, ApJ, 612, 1018-1025

[19] Tzeferacos, P., Ferrari, A., Mignone, A., et al. 2009, MNRAS, 400, 820 